\title{
Effect of Motherless Paternity Cases on the Interpretation of Parentage Investigations in a Population with Recurrent Inbreeding Practices
}

\section{Ansar El Andari ${ }^{1,2}$ and Issam Mansour ${ }^{1^{*}}$}

${ }^{1}$ Department of Laboratory Science and Technology, American University of Science and Technology, Lebanon

${ }^{2}$ School of Criminal Justice, University of Lausanne, Switzerland

*Corresponding author: Issam Mansour, American University of Science and Technology, Lebanon, Tel: 009611218716; E-mail: fhs@aust.edu.Ib

Received date: October 22, 2017; Accepted date: October 28, 2017; Published date: November 05, 2017

Copyright: () 2017 El Andari A, et al. This is an open-access article distributed under the terms of the Creative Commons Attribution License, which permits unrestricted use, distribution, and reproduction in any medium, provided the original author and source are credited.

\begin{abstract}
Currently, parentage testing relies mainly on the study of Short Tandem Repeats (STRs) DNA profiles. Parentage cases could be DUO (motherless/fatherless cases) or TRIO (where both parents' and child's DNA profiles are available). The absence of the DNA profile of one of the parents in DUO parentage testing increases the testing uncertainties. The Lebanese population is known to have a high rate of endogamous and consanguineous marriages, which challenges the power of discrimination of the DNA profiles among different individuals.

The present study aimed at evaluating the effect of motherless paternity cases on the interpretation of parentage investigations in the Lebanese population with recurrent inbreeding practices. 64 real DUO parentage exclusions and 15 real TRIO parentage exclusion cases were re-evaluated by assessing the possibility of false inclusions when decreasing DNA profile sizes or when simulating DUO cases from TRIO ones.

One case showed false positivity with 6 mismatches in its TRIO state, but only one mismatch in its DUO scenario. The probability of parentage (POP) obtained for this case after omitting the mismatch (a frequent inappropriate malpractice in some local laboratories) was $99.9 \%$. This simulated DUO case was cleared as exclusion when tested using DNA profile sizes of 24 STR markers. None of the other cases showed false positive results.
\end{abstract}

Keywords: Parentage testing; Consanguineous marriages; STR DNA profile; TRIO; Genotype

\section{Introduction}

Parentage testing determines whether or not an alleged individual is the biological parent of a given child. It provides a numerical proof of the likelihood of an alleged parent to be the biological parent of the child. If so, the child should share at least half of the DNA profile alleles with each of the biological parents. Later, statistical calculations are performed to test the hypothesis that an alleged parent is the biological parent against the hypothesis that an unknown individual, from the same ethnic background, is the biological parent, using commonly the Essen-Möller formula (or derivations). This entails evaluating a given number of STR systems to constitute the DNA profile of the involved individuals using allele frequencies for that ethnic background.

\section{Parentage cases could be either of two kinds:}

1. TRIO cases, where the child's and both parents' DNA profiles are available.

2. DUO cases (usually paternity cases), where the child's and only one parent's DNA profiles are available. The TRIO scenario minimizes the uncertainties in these interpretations whereas the absence of one of the parents, in DUO cases, weakens the power of the statistical calculations, introduces a source of uncertainty, and increases the likelihood of false paternity inclusions and inconclusive.
Lebanon is a relatively small country having a surface area of 10,952 $\mathrm{km} 2$ located on the Eastern shore of the Mediterranean Sea. The Lebanese population constituting of around 4,5 million inhabitants, is known to have high rates of endogamous and consanguineous marriages, which challenges the power of discrimination of the DNA profiles among different individuals and introduces an additional source of uncertainty. Consanguinity is defined as the union between two individuals that are closely related to an extent that they can be second cousins or closer. Indeed, Lebanon has relatively high consanguineous marriage rates mounting up to an average of $36 \%$, 2] in many areas.

In addition, the country is characterized by 18 unevenly distributed religious communities living in a relative geographic isolation. Due to this isolation, in addition to certain religious and cultural practices, marriages in Lebanon take place within the same religious community at an average rate of $88 \%$ [3].

The Lebanese population stratification could lead to erroneous parentage testing results, where the inbreeding practices could affect STR allele frequencies as well as the power of discrimination of a given STR DNA profile [4]. Indeed, endogamy and consanguinity shape the genetic structure of the population [5] where the likelihood of observing matching allele types among different individuals is high [6].

In addition to a loss in systems heterozygosity, in parentage testing when the involved individuals belong to the same subpopulation, falsely alleged parents are less likely to exhibit allele mismatches with the child's genotype, than when no population subdivision is present; Consequently, the potential of uncertainties in the interpretation of parentage disputes would increase. 
Moreover, Lebanon lacks legislation concerning parentage testing; Therefore, Lebanese relationship testing laboratories usually follow international standards of their choice as to the range of the cut off limit recommended for parentage positivity interpretations. This could differ among countries, laboratories and experts. For instance, many Lebanese testing laboratories follow the American Association of Blood Banking recommendations of parentage positivity for a POP of 99.9\%, while others follow the European recommendations for a POP of $99.999 \%[7,8]$.

Another important factor is that the Lebanese DNA testing laboratories currently have presented a remarkable increase in DUO paternity requests. Statistical calculations could be misinterpreted in case of underestimating the population subdivisions. Also, inappropriately practices in dealing with STR mutation cases could be observed in local practices, where the mutated system is wrongly omitted from the calculations in many of the cases.

The present study aims at evaluating the effect of motherless paternity cases on the interpretation of parentage investigations in the Lebanese population which is characterized to have high inbreeding practices, and therefore, the likelihood of observing matching allele types among different individuals could be high.

As part of ongoing research studies tackling uncertainties in consanguineous and endogamous populations in relationship testing, paternity exclusions were re-analyzed to evaluate possible false inclusions when decreasing the profile sizes (24/16/11 genetic systems) or simulating DUO from TRIO families.

\section{Methodology}

\section{Paternity exclusion analysis was conducted by simulating:}

1. "DUO" (alleged father and child) from "TRIO" (alleged father, mother, and child) paternity exclusions and

2. 11 markers DNA profile from 16 markers DNA profile paternity analysis (SGM vs. Identifier kits, respectively) in an attempt to assess the probability of false inclusion in these cases when dealt with different scenarios.
Data entry for 182 DNA profiles constituted of 16 STR markers (Identifier kit markers) was performed using in-house developed software, the Forensic Information Management System (FIMS) for data analysis. 18 real "TRIO" exclusion cases were used for assessing the probability of false inclusions occurrence upon simulating them into "DUO" (motherless) cases. Therefore, 18 DUO cases were simulated from these 18 real TRIO exclusion cases, in order to assess the probability of false inclusion where the DNA profile of one of the parents is missing for analysis.

In addition, 64 real DUO exclusion cases and 18 real TRIO exclusion cases were reanalyzed using different DNA profile size: 11 markers' DNA profile versus 16 Markers' DNA profile, also to test the probability of false inclusion when using limited STR markers. All the cases were tested using a prior probability of 0.5 and derivation of the Essen-Möller formula.

One exclusion case was re-tested using 24 systems by the combination of two commercial typing kits, PowerPlex 16 HS and PowerPlex" ESI 17 [9].

\section{Results}

None of the simulated cases showed false paternity positivity after simulating (i) different DNA profile sizes (11 vs 16 STR markers) and (ii) DUO from TRIO cases except for one interesting case, where the TRIO scenario showed six mismatches between the alleged father and the child, while showing only one mismatch in its simulated DUO scenario.

When the POP was calculated in this case, it showed a POP value of $99.9 \%$ without taking into consideration the mutation rate, and a POP value of $0 \%$ when taking into consideration the mutation rate (Tables 1 and 2). Most interestingly, when the DNA profiles sizes were increased from 16 to 24 systems in the DUO state, 5 mismatches were observed (Table 3), which provides a potential solution for the misinterpretation of the DUO-case.

\begin{tabular}{|l|l|l|l|l|l|}
\hline Variables & Alleged Father & Mother & Child & Maternal Shared Allele & Paternal Shared Allele \\
\hline D7S820 & $8-10$ & $8-12$ & $8-8$ & 8 & 8 \\
\hline D16S539 & $11-13$ & $11-12$ & $9-11$ & 11 & none \\
\hline D18S51 & $14-20$ & $15-19$ & $14-15$ & 15 & 14 \\
\hline CSF1PO & $10-11$ & $10-10$ & $10-11$ & 10 & 11 \\
\hline D2S1338 & $19-23$ & $20-25$ & $19-25$ & 25 & 19 \\
\hline Amel. & Male & Female & Male & - & - \\
\hline vWA & $16-17$ & $14-17$ & $17-17$ & 17 & 17 \\
\hline D21S11 & $27-30.2$ & $28-29$ & $28-29$ & 28 or 29 & none \\
\hline D13S317 & $8-11$ & $11-11$ & $11-11$ & 11 & 11 \\
\hline TPOX & $8-11$ & $8-8$ & $8-10$ & 8 & none \\
\hline FGA & $23-27$ & $21-23$ & $19-23$ & 23 & none \\
\hline
\end{tabular}


Citation: El Andari A, et al. (2017) Effect of Motherless Paternity Cases on the Interpretation of Parentage Investigations in a Population with Recurrent Inbreeding Practices. J Forensic Res 8: 395. doi:10.4172/2157-7145.1000395

Page 3 of 4

\begin{tabular}{|l|l|l|l|l|l|}
\hline D3S1358 & $17-19$ & $16-17$ & $15-17$ & 17 & none \\
\hline D19S433 & $13-14$ & $14-14$ & $12-14$ & 14 & none \\
\hline D8S1179 & $13-15$ & $12-13$ & $12-15$ & 12 & 15 \\
\hline D5S818 & $11-12$ & $10-11$ & $11-12$ & 11 & 12 \\
\hline THO1 & $6-9$ & $6-9.3$ & $6-9$ & 6 & 9 \\
\hline
\end{tabular}

Table 1: Trio case showing six mismatches between the alleged father and the child.

\begin{tabular}{|l|l|l|l|}
\hline Variables & Alleged Father & Child & Paternal Shared Allele \\
\hline D7S820 & $8-10$ & $8-8$ & 8 \\
\hline D16S539 & $11-13$ & $9-11$ & 11 \\
\hline D18S51 & $14-20$ & $14-15$ & 14 \\
\hline CSF1PO & $10-11$ & $10-11$ & 11 \\
\hline D2S1338 & $19-23$ & $19-25$ & 19 \\
\hline Amel & Male & Male & - \\
\hline VWA & $16-17$ & $17-17$ & 17 \\
\hline D21S11 & $27-30.2$ & $28-29$ & none \\
\hline D13S317 & $8-11$ & $11-11$ & 11 \\
\hline TPOX & $8-11$ & $8-10$ & 8 \\
\hline FGA & $23-27$ & $19-23$ & 23 \\
\hline D3S1358 & $17-19$ & $15-17$ & 17 \\
\hline D19S433 & $13-14$ & $12-14$ & 14 \\
\hline D8S1179 & $13-15$ & $12-15$ & 15 \\
\hline D5S818 & $11-12$ & $11-12$ & 11 or 12 \\
\hline THO1 & $6-9$ & $6-9$ & 6 or 9 \\
\hline
\end{tabular}

Table 2: Duo case simulated from the previous Trio family.

\begin{tabular}{|c|c|c|c|}
\hline Variables & Alleged Father & Child & Paternal Shared Allele \\
\hline D3S1358 & $17-19$ & $15-17$ & 17 \\
\hline D19S433 & $13-14$ & $12-14$ & 14 \\
\hline D2S1338 & $19-23$ & $19-25$ & 19 \\
\hline D16S539 & $11-13$ & $9-11$ & 11 \\
\hline D18S51 & $14-20$ & $14-15$ & 14 \\
\hline D1S1656 & $13-14$ & $13-19.3$ & 13 \\
\hline D10S1248 & $13-15$ & $13-15$ & 13 or 15 \\
\hline D2S441 & $10-11$ & $10-12$ & 10 \\
\hline THO1 & $6-9$ & $6-9.3$ & 6 \\
\hline vWA & $16-17$ & $17-17$ & 17 \\
\hline
\end{tabular}


Citation: El Andari A, et al. (2017) Effect of Motherless Paternity Cases on the Interpretation of Parentage Investigations in a Population with Recurrent Inbreeding Practices. J Forensic Res 8: 395. doi:10.4172/2157-7145.1000395

Page 4 of 4

\begin{tabular}{|l|l|l|l|}
\hline D21S11 & $27-30.2$ & $28-29$ & none \\
\hline D12S391 & $18.3-22$ & $18-20$ & none \\
\hline D8S1179 & $13-15$ & $12-15$ & 15 \\
\hline FGA & $23-27$ & $19-23$ & 23 \\
\hline SE33 & $19-21$ & $17-37$ & none \\
\hline Penta E & $11-19$ & $7-15$ & none \\
\hline D5S818 & $11-12$ & $11-12$ & 11 or 12 \\
\hline D13S317 & $8-11$ & $11-11$ & 11 \\
\hline D7S820 & $8-10$ & $8-8$ & 8 \\
\hline CSF1PO & $10-11$ & $10-11$ & 10 or 11 \\
\hline Penta D & $10-10$ & $9-13$ & none \\
\hline TPOX & $8-11$ & $8-10$ & 8 \\
\hline Amel. & Male & Male & - \\
\hline
\end{tabular}

Table 3: Duo case simulated from the previous Trio family, but tested with 24 instead of 16 STR markers [POP=99.95 \% (if omitting the mismatch)].

\section{Discussions and Conclusions}

The Lebanese population is characterized by having high rates of endogamy and consanguinity, a factor that challenges the power of discrimination of the DNA profiles among different individuals and introduces uncertainty in DNA interpretations. Despite this fact, Lebanon lacks legislation concerning parentage testing; which gives the choice to Lebanese relationship testing laboratories to follow international standards of their choice. These standards could differ among countries, laboratories and experts, and could not be appropriate or applicable to populations like the Lebanese population. For instance, many Lebanese testing laboratories currently follow the American Association of Blood Banking recommendations of parentage positivity for a POP of $99.9 \%$. Moreover, Lebanese DNA testing laboratories have experience a remarkable increase in DUO paternity requests, which could introduce in this case uncertainties in the statistical calculations of paternity disputes. In addition, many Lebanese testing laboratories inappropriately deal with STR mutation cases where the testing laboratory simply omits the mutated system from the calculations in many of the cases.

The present study aimed at evaluating the effect of motherless paternity cases on the interpretation of parentage investigations in the Lebanese population with recurrent inbreeding practices where the likelihood of observing matching allele types among different individuals is high.

DUO simulations of TRIO paternity exclusions showed one case of false paternity inclusion using 16 STR markers when omitting the mismatch, which was then resolved by retesting the case using 24 STR markers. From assessing these results and the genetic structure of the Lebanese population, we consequently recommend the use of TRIO analysis in parentage tests performed in this population, or the increase in the DNA profile size for the evaluation of any DUO parentage dispute.

\section{Acknowledgement}

Authors would like to thank the anonymous reviewers for their constructive and useful comments. Promega corporation for granting this project.

\section{References}

1. Kanaan ZM, Mahfouz R, Tamim H (2008) The prevalence of consanguineous marriages in an underserved area in lebanon and its association with congenital anomalies. Genet Test 12:367-372.

2. Barbour B, Salemeh P (2009) Consanguinity in lebanon: Prevalence, distribution and determinants. J Biosoc Sci 41: 505-517.

3. Klat M, Khudr A (1986) Religious endogamy and consanguinity in marriage patterns in Beirut, Lebanon. Biodemography Soc Biol 33: 138-145.

4. Andari AE, Othman H, Taroni f, Mansour I (2013) Population genetic data for 23 STR markers from Lebanon. 7: 108-113.

5. Mansour I, Delague V, Cazeneuve C, Dode C, Chouery E, et al. (2001) Familial mediterranean fever in lebanon: Mutationspectrum, evidence for cases in maronites, greek orthodoxes, greek catholics, syriacs and chiites and for an association between amyloidosis and M694V and M694I mutations. Eur J Hum Genet 9: 51-55.

6. Ayres K (2000) Relatedness testing in subdivides populations. Forensic Sci Int 114: 107-115.

7. American association of blood banks(AABB) (2009) Standards for relationship testing laboratories, 9 th edtn.

8. Noel P (1976) Journal des tribunaux, droit federal.

9. Promega corporation (1978) Madison, USA. 